\title{
Hands on LUNA/DarkSide: Silicon photomultiplier using a Plastic Scintillator
}

\section{Simone Copello*}

Dipartimento di Fisica, Universitá degli studi di Genova, Genova, Italy

E-mail: scopello@ge.infn.it

\section{Andreas Best}

INFN - Laboratori Nazionali del Gran Sasso, Assergi, Italy

E-mail: andreas.bestalngs.infn.it

\author{
Alessandro Razeto \\ INFN - Laboratori Nazionali del Gran Sasso, Assergi, Italy \\ E-mail: alessandro.razeto@lngs.infn.it
}

\begin{abstract}
The usage of scintillating materials is one of the most common techniques for particle detection. Photomultiplier tubes (PMT) are often used to reveal light signals, but they present some technical difficulties as low integration level, high voltage power supply and magnetic fields sensitivity. One alternative is represented by photodiodes, as silicon photomultipliers (SiPM), which are more compact, have higher quantum efficiency and low power consumption. On the other hand they have high dark rate (i.e. higher noise) and small active surface. During my activity in Gran Sasso Summer Institute I acquired gamma spectra using a plastic scintillator to make a comparison between performances of PMT and SiPM.
\end{abstract}

Gran Sasso Summer Institute 2014 Hands-On Experimental Underground Physics at LNGS - GSSI14, 22 September - 03 October 2014

INFN - Laboratori Nazionali del Gran Sasso, Assergi, Italy

\footnotetext{
* Speaker.
} 


\section{Use of PMT in DarkSide and LUNA experiments}

LUNA (Laboratory for Underground Nuclear Astrophysics) and DarkSide are both at Laboratori Nazionali del Gran Sasso, Italy. The first one is a laboratory for nuclear astrophysics which uses an ion accelerator to study cross sections, while the second one is an experiment for direct dark matter detection using a two-phase liquid argon time projection chamber. Both the experimental setups use photomultiplier tubes (PMT) to detect light produced by scintillation (of liquid Argon or Bismuth Germanate cristal).

PMTs are the most widely used devices to convert weak light signals, typically no more than few hundred photons, into current pulses and represent a well known technology but their usage implies some technical complications. For this reasons advances in semiconductor technology have led to the substitution of solid-state devices. In general, photodiodes offer the advantages of higher quantum efficiency, lower power consumption, more compact size and are insensitive to magnetic fields. their working principle is based on the fact that when light is incident on a semiconductor, with photons of a typical energy of 3-4 eV, it creates electron-hole pairs. In particular, in the case of avalanche photodiodes, the small amount of produced charge is increased by the avalanche effect that occurs at high values of applied voltage, about tens of volts [4]. On the other hand they present some disadvantages, which are a very high dark rate (the frequency a thermal fluctuation causes a fake single photon signal) and a small active surface: few $\mathrm{cm}^{2}$ compared to hundreds of $\mathrm{cm}^{2}$. A possible solution to the first problem is to reduce the working temperature to cryogenic temperatures, this is one of the present $R \& D$ activity goals.

My activity during the Gran Sasso Summer Institute was make a comparison between the particle detection obtained using a plastic scintillator with a PMT (model?) and a silicon photomultiplier (SiPM) (model: ASD-RGB-SiPM4S-P).

\section{Experimental setup}

I could choose between three different plastic scintillators, whose characteristics are visible in table 1, to build the setup. In particular I choose the BC-408 because of its high light yield. Its dimensions were $6.5 \times 6.5 \times 5 \mathrm{~cm}^{3}$. It was enclosed into a stainless-steel box between the PMT and the SiPM. Power supply and read out signal go out through apposite connectors on the short sides of the box (see figure 1). Most of the measurements have been performed using the aluminium wrapped scintillator, but, making a direct comparison, the foil seemed not to reflect a considerable fraction of light.

Before using the scintillator, I estimated the characterization curve of the PMT, studying the relationship between gain and high voltage (HV). To do this I acquired several spectra using different $\mathrm{HV}$ and I fitted the single photon peak. The same procedure is unfeasible for the SiPM because of the pile-up due to the high dark rate of the device. Basing on this I choose the value $H V=1440 \mathrm{~V}$ that corresponds to $12.2 \mathrm{pV} \cdot \mathrm{s} /$ photon. Spectra were obtained acquiring the PMT signal with a digital oscilloscope, using a threshold trigger and computing the baseline integral over a fixed time interval. 


\begin{tabular}{|c|c|c|c|c|c|}
\hline Model & Light output & $t_{\text {rise }}(n s)$ & $t_{\text {decay }}(n s)$ & attenuation length $(\mathrm{cm})$ & $\lambda_{\max }(\mathrm{nm})$ \\
\hline BC 408 & $64 \%$ & 0.9 & 2.1 & 210 & 425 \\
BC 428 & $36 \%$ & 1.6 & 12.5 & 150 & 480 \\
BC 430 & $45 \%$ & 3.2 & 16.2 & - & 580 \\
\hline
\end{tabular}

Table 1: Characteristics of the three plastic scintillators from "Saint-Gobain" I had available. Light output is expressed as fraction of anthracene light yield, which is 17400 photons $/ \mathrm{MeV}$. Datas from producer datasheets ([1] [2] [3]).
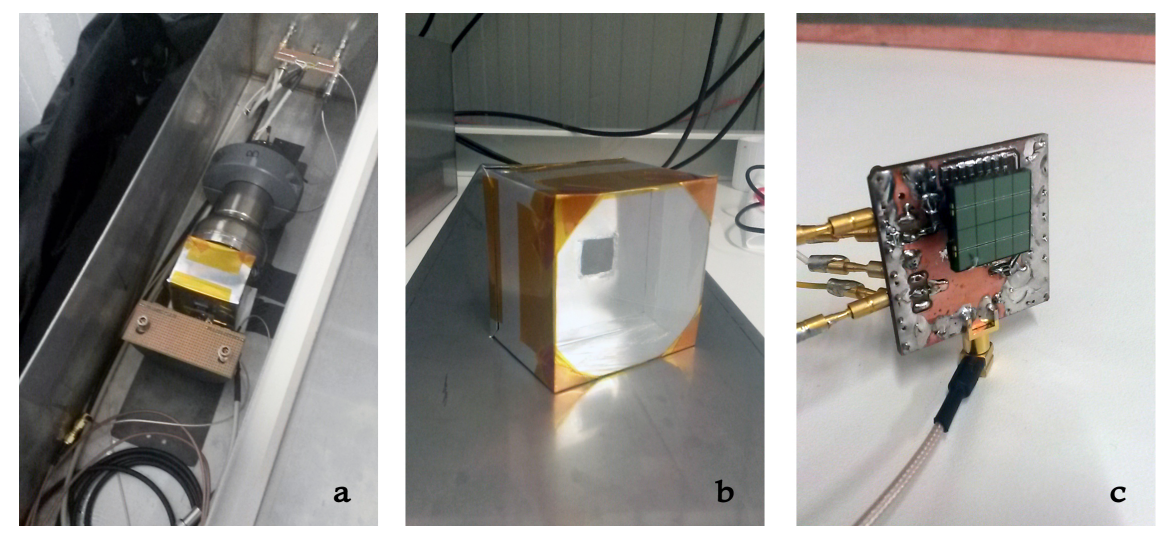

Figure 1: Steel box (a) containing, from top to bottom, PMT, plastic scintillator BC-408 wrapped with aluminium foil (b) and SiPM with its amplification electronics (c).

\section{Measurement and analysis}

I tried to estimate the collection efficiency $E_{c}$ of the system composed of scintillator and PMT (including geometrical acceptance, reflection and trasmission efficiency on the interfaces and the scintillator auto-absorption). Known parameters were light yield ( $L_{y} \simeq 11070$ photons $\left./ \mathrm{MeV}\right)$ and quantum efficiency (in average $\simeq 70 \%$ ).

To do this I wrote a toy Monte Carlo to reproduce the signal of atmospheric muons acquired by the PMT. The software works as follows. It generates the muon trajectory from a random point in a plane above the scintillator, using a zenith angle following the $\cos ^{2}(\theta)$ distribution. It then evaluates the path length inside the volume and the energy released by the particle, assumed to be minimum-ionizing. The average number of produced photons is multiplied by the collection efficiency $E_{c}$ and the obtained value is used to extract the final number of photons incident on the photocathode $\left(N_{c}\right)$ from a poissonian distribution with that mean value. Finally, to reproduce the intrinsic resolution of the PMT, the real number of detected photons is extracted from a gaussian function having mean value $N_{c}$ and $\sigma \propto N_{c}$. In figure 2 the result is shown, using $E_{c}=0.071$ and $\sigma / E=0.06$. Taking into account the ratio between PMT and SiPM active surfaces, we can conclude that the collection efficiency of the SiPM in this setup is about 7.1\%/25 $=0.28 \%$.

In order to do a direct comparison between PMT and SiPM spectra I used several gamma sources $\left({ }^{22} \mathrm{Na},{ }^{60} \mathrm{Co},{ }^{133} \mathrm{Ba}\right.$ and $\left.{ }^{137} \mathrm{Cs}\right)$. But not in all the cases the acquired spectrum was useful because the peak reconstruction was not feasible. In particular, spectra from PMT were fitted using a gaussian function plus an exponential background, while the SiPM ones were fitted using two 

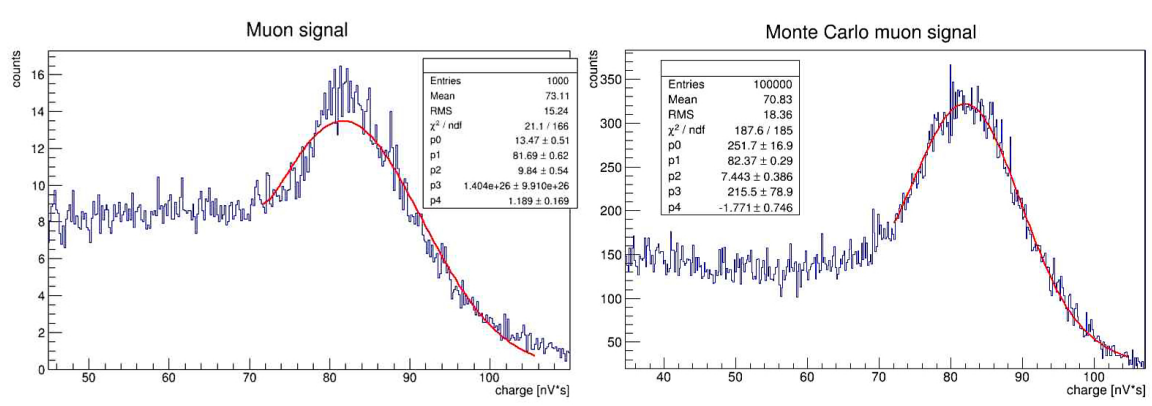

Figure 2: Comparison between muon signal spectrum from PMT and the simulated one, obtained using collection efficiency $E_{c}=7.1 \%$ and $\sigma / E=0.06$.

gaussian functions, one for the peak and one for the background (produced by the baseline noise). Some of these fits are shown in figure 3. In addition to this I also acquired some spectra using a small Bismuth Germanate crystal (BGO), applying the same peak identification method. All the results are summarized in figure 4.

\section{Conclusions}

Taking into account the analysis of muons datas, the collection efficiency of $7.1 \%$ appears a bit smaller than the expected value and, considering the system geometry, aluminium foil seems the to be useless. The reflecting coating and the optical coupling between scintillator and sensors could be improved. The estimated efficiency value is however consistent with datas of gamma peaks since the peaks actually are in correspondence of the expected number of photons.

For PMT signals $\sigma$ of the peaks is two to four times bigger than expected if we just consider the poissonian distribution of the collected number of photons. For example the ${ }^{22} \mathrm{Na}$ peaks, at 1270 $\mathrm{keV}$, shows an average value $N_{\gamma} \simeq 700 \gamma$ and $\sigma_{\gamma} \simeq 85 \gamma$ which means a ratio $\sigma_{\gamma} / \sqrt{N_{\gamma}} \simeq 3.2$. For SiPM signals is $N_{\gamma} / \sigma_{\gamma} \simeq 5$ and this is probably due to the thermal noise of the device, which is bigger in SiPM than in PMT.

\section{References}

[1] Saint-Gobain datasheet "BC-400/BC-404/BC-408/BC-412/BC-416 Premium Plastic Scintillators" bc400-416.pdf

[2] Saint-Gobain datasheet ”BC-428, Green-Emitting Plastic Scintillators" bc428.pfd

[3] Saint-Gobain datasheet ”BC-430, Red-Emitting Plastic Scintillators” bc430.pdf

[4] Glenn F. Knoll "Radiation detection and measurement” 

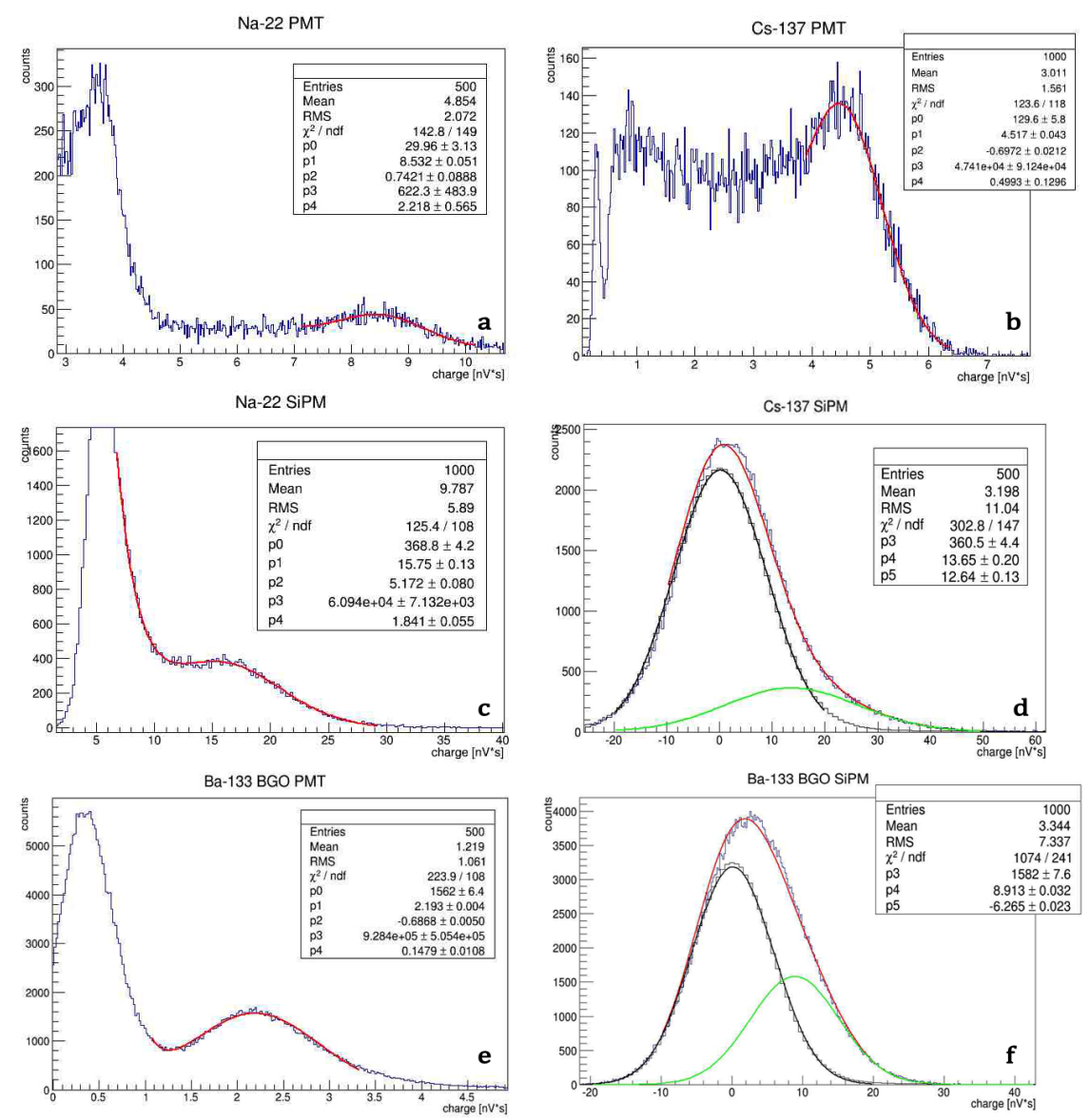

Figure 3: Some of the fitted peaks. Two from PMT: ${ }^{22} \mathrm{Na}$ at $1274 \mathrm{keV}$ (a) and ${ }^{137} \mathrm{Cs}$ at $662 \mathrm{keV}$ (b). Two from SiPM: ${ }^{22} \mathrm{Na}$ at energy between 511 and $1274 \mathrm{keV}$ because the resolution is not enough to distinguish the peaks (c) and ${ }^{137} \mathrm{Cs}$ at $662 \mathrm{keV}(\mathrm{d})$. Two were obtained using BGO crystal: ${ }^{133} \mathrm{Ba}$ at $\sim 350 \mathrm{keV}$ with PMT (e) and with SiPM (f).
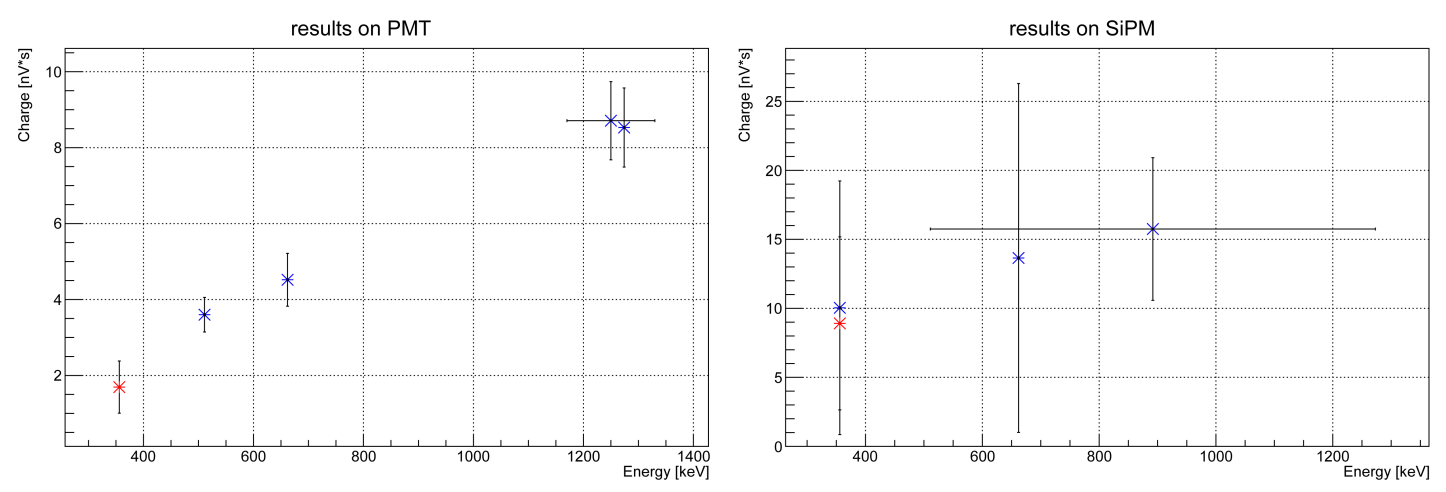

Figure 4: Here the results are shown, blue dots represent spectra from plastic scintillator BC-408, while red dots represent spectra from BGO crystal. In the left plot we can see values obtained from PMT: from left to right, ${ }^{133} \mathrm{Ba}(\sim 350 \mathrm{keV}),{ }^{22} \mathrm{Na}(511 \mathrm{keV}),{ }^{137} \mathrm{Cs}(662 \mathrm{keV}),{ }^{60} \mathrm{Co}(1250 \mathrm{keV})$ and ${ }^{22} \mathrm{Na}(1270 \mathrm{keV})$. The right plot contains results from SiPM: ${ }^{133} \mathrm{Ba},{ }^{137} \mathrm{Cs}$ and ${ }^{22} \mathrm{Na}$. 